\title{
La Bretagne des origines à nos jours
}

Jean-Claude Meuret

\section{OpenEdition}

Journals

Édition électronique

URL : http://journals.openedition.org/rao/963

DOI : $10.4000 /$ rao.963

ISBN : 978-2-7535-1609-0

ISSN : 1775-3732

Éditeur

Presses universitaires de Rennes

Édition imprimée

Date de publication : 31 décembre 2009

Pagination : 223-224

ISBN : 978-2-7535-1086-9

ISSN : 0767-709X

\section{Référence électronique}

Jean-Claude Meuret, «La Bretagne des origines à nos jours », Revue archéologique de l'Ouest [En ligne],

26 | 2009, mis en ligne le 31 décembre 2011, consulté le 03 décembre 2020. URL : http://

journals.openedition.org/rao/963 ; DOI : https://doi.org/10.4000/rao.963 
plus récentes, alliant à la fois les données événementielles ou politiques et les faits de société. Il faut souligner le souci de l'auteur d'intégrer toutes les données sélectionnées dans le temps long. C'est ainsi que le rattachement de la Bretagne à la France n'est pas présenté comme une rupture brutale ou un viol identitaire; elle est au contraire dédramatisée et inscrite dans la réalité des faits historiques, ne serait-ce que la conservation de certains " privilèges » telle l'exemption de gabelle. De manière générale, sont suggérés avec justesse les grands mouvements de balancier qu'a connus la Bretagne, le plus souvent différents, voire à contre-temps de ceux du reste de la France : guerre de Cent ans moins sensible que dans le royaume, guerres de religion du XVI ${ }^{\mathrm{e}}$ siècle plus brèves, " âge d'or " au XVI ${ }^{\mathrm{e}}$ mais appauvrissement au XVIII ${ }^{\mathrm{e}}$ siècle, puis absence d'industrialisation au XIx ${ }^{e}$. Se dégage ainsi la grande spécificité de la Bretagne : région péninsulaire, sa prospérité implique nécessairement des relations maritimes ouvertes; le fait est d'ailleurs observé depuis les âges du Bronze et du Fer.

Pour la Révolution de 1789, l'accent est mis sur le rôle moteur tenu par certains Bretons, ce que le grand public ne sait pas toujours. En venant au XIX ${ }^{e}$ siècle, l'auteur décrit une Bretagne surpeuplée et seulement agricole, une Bretagne terre d'émigration vers la capitale, une Bretagne réticente à l'égard des nouveaux régimes politiques, y compris la III ${ }^{e}$ République dont la mise en place s'accompagne d'un formidable mouvement de reconstruction d'églises, véritable manifeste politique et religieux affiché dans la pierre.

L'auteur souligne aussi la terrible saignée humaine de la guerre 1914-18, plus forte ici qu'ailleurs et qui contribua encore à la stagnation de la région. À compter des années 1950, l'émergence et le rôle du CELIB sont bien mis en lumière pour éclairer le décollage de la Bretagne. C'est sur ce fond dynamique que la région connaît alors un remarquable essor agricole, industriel et culturel et cesse d'être terre d'émigration depuis les années 1970.

Il est facile d'exprimer certaines absences dans un ouvrage limité en volume et donc forcément sélectif. On a déjà noté la trop faible place faite à l'apport de l'archéologie à l'histoire, on regrettera aussi le peu de place accordé à la Haute Bretagne dans ce tableau discutable d'une région monolingue et monoculturelle; on aurait aussi aimé voir abordé par l'historien le problème et l'expression galvaudée et contestable de «Bretagne historique ». Et puis on attendrait quelques lignes sur cette carte de la Bretagne actuelle (p. 124) où la majorité des activités industrielles se situent à l'est du méridien de Rennes. Mais s'il n'est fait aucune place à ces questions, tout comme au manoir, à la ferme, à la musique, au costume, aux mythes... bretons, c'est que B. Merdrignac souhaitait avant tout se concentrer sur l'essentiel, éviter les idées reçues et transmettre un certain nombre d'analyses nouvelles. Pour cela, il s'est toujours placé sur et dans le fil événementiel de l'histoire bretonne, afin de replacer celle-ci dans le temps long et transmettre au public large une synthèse, non une analyse détaillée.

Jean-Claude Meuret

Collectif, 2007 - «Découvertes archéologiques récentes dans l'Orne », Bulletin trimestriel de la Société historique et archéologique de l'Orne, 126, $3^{\mathrm{e}}$ et $4^{\mathrm{e}}$ trimestres (septembre-décembre 2007), 199 p. (ISSN 0154-0505, $20 €$ ).

Ce volume formant la seconde partie de la cent vingtsixième livraison du Bulletin de la Société historique et archéologique de l'Orne regroupe en quelque deux cents pages onze articles dédiés à un panorama des recherches archéologiques récemment conduites dans le département ornais, précédés d'une préface du Conservateur régional de l'Archéologie de Basse-Normandie. L'ensemble témoigne du dynamisme de l'archéologie, notamment (mais pas seulement) préventive, dans ce département bas-normand, jusqu'alors relativement peu concerné au regard de ses voisins de la Manche et du Calvados.

En ouverture, E. Ghesquière consacre un article liminaire aux découvertes " multi périodes" réalisées dans le cadre du diagnostic de la future autoroute A 88 entre Falaise et Argentan. Les résultats exposés concernent un large segment chronologique, du Néolithique au Moyen Âge. Les plans des sites et vestiges sont présentés avec le mobilier d'accompagnement et une notice synthétique. La communication suivante (F. Morand et al.) décline les acquis d'une campagne de prospections en forêt de Bellême, vaste ensemble forestier totalisant aujourd'hui 2400 ha aux marches sud de la Normandie historique, au sein duquel ont été recensés divers sites de type enceinte quadrangulaire, tertre, chemin, plate-forme, fossé et/ou parcellaire, carrière, traces de la seconde Guerre mondiale. Chacune de ces catégories donne lieu à un exposé détaillé; s'y ajoute une dernière partie due à N. Zaour, récapitulant les principales caractéristiques des déchets, structures et témoins d'activités métallurgiques qui abondent dans cette partie du Perche (préparation et réduction de minerai de fer). 
Les contributions qui suivent respectent un fil chronologique et sont dédiés à des sites ou aspects archéologiques plus ciblés. D. Cliquet et al. ont ainsi consacré un premier développement à l'étude des ateliers de bifaces de «l'espace d'Écouché ", âgés d'environ 600 ans. Ils mettent à profit cette opportunité pour aborder le problème de l'identification des peuplements néandertaliens et aurignaciens dans la région normande et le Grand-Ouest; il semble que les preuves de la présence de l'Homme moderne se fassent encore attendre dans le département de l'Orne, alors qu'elles ont déjà été détectées dans les régions limitrophes. Cyril Marcigny et al. nous livrent ensuite les principaux résultats d'un diagnostic d'archéologie préventive conduit sur le site de hauteur de l'éperon d'Exmes, sur la place de l'église dont les origines, jadis étudiées par l'archéologue orientaliste du Mesnil du Buisson, remontent bien au-delà du Moyen Âge. Renouant avec les découvertes antérieures, les auteurs proposent une vision renouvelée de la succession des occupations, du Paléolithique à l'ère contemporaine. Les vestiges les plus importants concernent les niveaux de La Tène finale et de l'époque augustéenne, correspondant à l'occupation d'un vaste oppidum dont le tracé est identifié au sommet de l'éperon. Puis les $\mathrm{IV}^{\mathrm{e}}-\mathrm{VI}^{\mathrm{e}}$ siècles voient le développement présumé d'un complexe paléochrétien accueillant, peut-être au $v^{e}$ siècle, deux églises jumelles encadrant un parvis central, l'une étant située juste en dessous du sanctuaire paroissial. Cette disposition caractéristique des premiers ensembles cathédraux serait fort susceptible de coïncider avec les attestations écrites faisant état, entre 511 et 538, dans les listes épiscopales de la province de Rouen, de deux évêques d'Hiémois nommés Litardus et Passivus. Rappelons que dès 541, ce dernier est explicitement mentionné comme évêque de Sées, ce qui conduit à envisager le déplacement - définitif du siège épiscopal en faveur de cette capitale entre 538 et 541. Nonobstant les importants compléments apportés par cette intervention aux premiers scénarios élaborés par du Mesnil du Buisson dans les années quarante, ces découvertes appellent d'autres investigations propres à définir précisément la chronologie du rempart de l'oppidum.

À la suite, F. Delrieu produit un exposé complet sur l'histoire du Camp de Bierre, à Merri, " l'un des seuls sites de hauteur fortifiés à avoir suscité l'intérêt des archéologues ornais dès le milieu du XviII ${ }^{e}$ siècle ». Après un récapitulatif des données existantes sont présentés les derniers résultats glanés à l'occasion de sondages programmés conduits par l'auteur, qui conclut sur une mise en perspective dans le contexte régional des $\mathrm{IX}^{\mathrm{e}}-\mathrm{VI}^{\mathrm{e}}$ siècles av. J.-C. On notera cependant que ce contexte ne recouvre pas la totalité de l'histoire du site puisque l'ultime phase de relèvement du rempart du Camp de Bierre intervient au $\mathrm{IX}^{\mathrm{e}}$ ou $\mathrm{X}^{\mathrm{e}}$ siècle après J.-C., comme l'avaient déjà clairement signalé
G. Guillemot et A. Chancerel dans une précédente livraison du Bulletin : un pan plutôt méconnu de l'histoire médiévale de la Normandie sur lequel il serait sans doute utile de se pencher davantage.

Les articles suivants concernent l'époque gauloise et galloromaine, avec tout d'abord une présentation synthétique du sanctuaire du "Pré du Mesnil », à Aunou-sur-Orne, par T. Lejars et L. Perret, site fréquenté de La Tène moyenne au $\mathrm{III}^{\mathrm{e}}$ siècle après J.-C. L'accent est surtout mis sur l'analyse du mobilier dans un contexte cultuel, non dénué d'aspects " exotiques " puisqu'on y dénombre notamment un mors bimétallique de tradition thrace. C.-C. Besnard Vauterin et al. décrivent ensuite un petit habitat enclos de la fin de La Tène accompagné de deux sépultures à mobilier (dont le corps d'une femme assassinée, son crâne arborant une spectaculaire lésion causée par un objet tranchant), auquel a succédé un fanum du Haut-Empire à Nécy, sur le tracé de l'autoroute A88. Ce petit sanctuaire rural, probablement lié au passage de la voie reliant les chefs-lieux de Sées et Vieux, n'est manifestement plus fréquenté au-delà de la seconde moitié $\mathrm{du} \mathrm{II}^{\mathrm{e}}$ siècle après J.-C. Le thème cultuel est encore abordé par G. Leclerc à travers une autre présentation synthétique du site gallo-romain de Macé, interprété comme un "sanctuaire complexe " réunissant un fanum principal et plusieurs temples et édicules, parmi d'autres structures (fosses, foyers), au sein d'une aire sacrée commune. La minutieuse fouille programmée conduite sur ce site peu commun a permis de distinguer quatre phases successives, marquées par des réaménagements complexes. La période de fréquentation s'inscrit entre le début de l'époque augustéenne et les années 360-370, ce qui conduit l'auteur à envisager un lien de causalité entre la fondation du sanctuaire et les prémices urbaines de la ville antique de Sées d'une part, son abandon et les débuts de la christianisation dans l'Orne d'autre part.

Retour sur le tracé de l'A 88 avec l'article suivant, dû à L. Le Gaillard qui nous livre les principaux acquis de la fouille d'une villa gallo-romaine à Marcei. Cet établissement d'envergure, comparable à d'autres grandes villae de la région normande, connaît une évolution importante au cours du Haut et du Bas Empire, traduite par trois états successifs; la dernière phase d'occupation manifeste cependant un déclin notable. Arguant de l'exiguïté du décapage (lié à l'emprise autoroutière), l'auteur conclut un peu curieusement sur une discussion autour de ses incertitudes quant à l'identification définitive du site en tant que villa, au détriment de l'hypothèse d'un sanctuaire, et préconise le recours à des investigations plus étendues. La fouille de Marcei laisse place à un nouveau diagnostic, conduit à Sées par le même auteur et à l'occasion duquel ont été mis au jour et analysés divers témoins d'habitat et d'activité métallurgique remontant au $\mathrm{I}^{\text {er }}$ siècle après J.-C. L'histoire du chef-lieu sagien est 
évoquée à partir d'une cartographie minutieuse des vestiges antiques, médiévaux et modernes recensés à ce jour, puis les vestiges sont présentés en détail, intégrant études de mobilier (céramique) et de déchets métallurgiques. Les acquis de cette opération sont pour finir, replacés dans le contexte général de l'histoire du cœur urbain de Sées.

La dernière contribution concerne le Moyen Âge avec une étude d'archéologie du bâti dédiée à la "Maison des Sept Colonnes ", imposant édifice à pans de bois construit sur quatre niveaux en plein centre de la ville médiévale d'Alençon, travail mené par J.-D. Desforges dans le cadre d'un important projet de restauration. L'histoire détaillée de cet édifice inscrit à l'Inventaire du Patrimoine est relatée depuis ses origines lointaines ( $\mathrm{XI}^{\mathrm{e}}-\mathrm{XII}^{\mathrm{e}}$ siècles), et agrémentée d'une riche iconographie, avant d'aboutir aux schémas de restitution des façades, aux plans des caves et du rez-de-chaussée.

On ne sait au juste si cet ouvrage contribuera effectivement, ainsi que l'annonce le préfacier, à " forger l'Orne du $\mathrm{xxI}^{\mathrm{e}}$ siècle ». Peu importe en définitive, car les actualités qu’il contient offrent un réel intérêt non seulement pour la fratrie des érudits départementaux, mais aussi pour l'ensemble de la communauté historienne et archéologue régionale. On peut seulement déplorer, outre le choix un " tantinet » austère de la couverture au regard d'une belle iconographie en couleurs insérée dans le volume (un à-plat terreux surplombé par quelques gravats et deux sacs poubelle, pour évoquer le majestueux Camp de Bierre!), le caractère éphémère de certaines données (diagnostics), ou encore la brièveté de certaines analyses portant sur des thématiques d'actualité qui requièrent, dans l'avenir, de plus amples développements. En bref, une lecture utile pour prendre la mesure des recherches archéologiques en cours dans ce secteur encore méconnu mais assurément prometteur de la Basse-Normandie.

Vincent Carpentier

INRAP / CRAHAM-Centre Michel-de-Boüard, FRE (UCBN-CNRS) n 3119

Collectif, 2009 - Revue archéologique d'Île-de-France, 1 (2008), 424 p. (www.raif.fr; $15 €$ ).

C'est toujours un bonheur que d'avoir à saluer l'apparition d'une nouvelle revue dans le " paysage archéologique français ", surtout en des temps où les tirages tendent à se tasser et où la situation financière de plus d'un titre s'avère délicate.

On pourrait se demander si le tissu des revues interrégionales souhaité par le Ministère de la Culture pour présenter les résultats de l'archéologie nationale en complément des revues locales et départementales est bien compatible avec l'émergence d'un titre à vocation régionale. Ce serait oublier que toute planification rigide est incompatible avec la liberté d'esprit qui s'impose en matière de recherche; d'ailleurs, la région Île-de-France semble suffisamment riche en vestiges (avérés ou potentiels), suffisamment active économiquement (avec toutes les opérations préventives que cela implique) et suffisamment dotée humainement comme institutionnellement pour fournir la matière à une publication spécifique.

L'idée d'une telle revue n'était pas nouvelle comme le rappelle dans sa préface le Conservateur régional de l'Archéologie, mais nul doute que la passe délicate que traverse la Revue archéologique du Centre et de l'Île-de-France, héritière en 1995 de la vieille $R A C F$, ait incité l'association Les Amis de la Revue archéologique d'île-de-France à "sauter le pas ". Espérons simplement que la similitude des sigles (RAIF / RACIF) ne crée pas trop de confusion et ne contribue pas à marginaliser un support de publication indispensable pour tout le centre de la France, surtout à l'heure où le lectorat des particuliers tend à se contracter et où plus d'une bibliothèque se voit contrainte de remettre en question ses abonnements.

Le parti d'une revue "généraliste " a été retenu, ce qu'il convient de saluer en des temps où chacun est parfois tenté de s'enfermer dans sa micro-spécialité et, sous l'avalanche des publications, de ne considérer que ce qui colle au plus près de ses interrogations immédiates. Saluons donc le sommaire riche et équilibré de ce volume inaugural de 424 pages : vingt articles, d'ampleur très variable (de 6 à 52 pages) et regroupent plus de quarante signatures et couvrent toutes les périodes, du Paléolithique supérieur au début du $\mathrm{Xx}^{\mathrm{e}}$ siècle, où l'on passe d'une vieille découverte du XVII ${ }^{\mathrm{e}}$ siècle "revisitée " à une actualité encore chaude. Il n'est pas possible de faire ici l'analyse détaillée de chaque contribution, toutes étant de très bonne tenue scientifique; disons seulement que l'on est frappé, en feuilletant la revue, de voir que pratiquement tous les articles traitent de découvertes ou d'opérations ponctuelles, même si le sujet est - plus ou moins - élargi dans la discussion. On pourrait croire à un effet " premier numéro ", chaque auteur pressenti ayant fourni le papier qu'il avait plus ou moins sous la main, mais la maquette (avec, en tête d'article, une carte régionale où le département concerné est grisé et le site pointé) laisse présumer un parti rédactionnel, pas si éloigné de celui des 